\title{
MicroRNA-345 induces apoptosis in pancreatic cancer cells through potentiation of caspase-dependent and -independent pathways
}

\author{
Sanjeev K Srivastava ${ }^{1,4}$, Arun Bhardwaj ${ }^{1,4}$, Sumit Arora ${ }^{1}$, Nikhil Tyagi ${ }^{1}$, Seema Singh ${ }^{1}$, Joel Andrews ${ }^{1}$, \\ Steve McClellan ${ }^{1}$, Bin Wang ${ }^{2}$ and Ajay P Singh ${ }^{\star, 1,3}$ \\ ${ }^{1}$ Department of Oncologic Sciences, Mitchell Cancer Institute, University of South Alabama, 1660 Springhill Avenue, Mobile, \\ AL 36604-1405, USA; ${ }^{2}$ Department of Mathematics and Statistics, University of South Alabama, Mobile, AL, USA and ${ }^{3}$ Department \\ of Biochemistry and Molecular Biology, College of Medicine, University of South Alabama, Mobile, AL, USA
}

Background: Previously, miR-345 was identified as one of the most significantly downregulated microRNAs in pancreatic cancer $(\mathrm{PC})$; however, its functional significance remained unexplored.

Methods: miR-345 was overexpressed in PC cells by stable transfection, and its effect on growth, apoptosis and mitochondrialmembrane potential was examined by WST-1, Hoechst-33342/Annexin-V, and JC-1 staining, respectively. Gene expression was examined by quantitative reverse-transcription-PCR and/or immunoblotting, and subcellular fractions prepared and caspase-3/7 activity determined by commercially available kits. miR-345 target validation was performed by mutational analysis and luciferasereporter assay.

Results: miR-345 is significantly downregulated in PC tissues and cell lines relative to normal pancreatic cells, and its expression decreases gradually in PC progression model cell lines. Forced expression of miR-345 results in reduced growth of PC cells because of the induction of apoptosis, accompanied by a loss in mitochondrial membrane potential, cytochrome-c release, caspases-3/7 activation, and PARP-1 cleavage, as well as mitochondrial-to-nuclear translocation of apoptosis-inducing factor. These effects could be reversed by the treatment of miR-345-overexpressing PC cells with anti-miR-345 oligonucleotides. BCL2 was characterised as a novel target of miR-345 and its forced-expression abrogated the effects of miR-345 in PC cells.

Conclusions: miR-345 downregulation confers apoptosis resistance to PC cells, and its restoration could be exploited for therapeutic benefit.

Pancreatic cancer (PC) is one of the deadliest malignancies. With an estimated 48960 diagnoses and nearly 40560 deaths in 2015, it is considered the fourth leading cause of cancer-related death in the United States (Siegel et al, 2015). Owing to the lack of specific symptoms, early detection is not possible and most PCs are diagnosed in advanced and metastatic stage, leaving limited options for therapy (Feldmann and Maitra, 2008). Most current therapies provide no or marginal survival benefits and therefore 5-year overall survival rate of PC patients has stayed at 3-6\% for past few decades (Arora et al, 2013). Clearly, there is an urgent need to identify novel diagnostic/therapeutic targets, understand the molecular mechanisms underlying the aggressive nature of this

\footnotetext{
*Correspondence: Dr AP Singh; E-mail: asingh@health.southalabama.edu

${ }^{4}$ These authors contributed equally to this work.
}

Received 29 April 2015; revised 10 June 2015; accepted 13 June 2015; published online 6 August 2015 
malignancy, and develop alternative strategies and novel therapeutics for effective treatment of this devastating disease.

MicroRNAs (miRNAs) are small ( $\sim 22$-nucleotides) noncoding RNAs that act as important regulators of gene expression (Bhardwaj et al, 2010; Srivastava et al, 2013). miRNAs act by specifically binding to the $3^{\prime}$ untranslated region (UTR) of their cognate target mRNAs and subsequently cause either their degradation or inhibit translation (Bhardwaj et al, 2010). To date, $\sim 2469$ miRNAs have been identified in humans (Friedlander et al, 2014; Zearo et al, 2014), and emerging evidence suggests that miRNAs potentially regulate $\sim 30-80 \%$ of all protein-coding genes (Lu and Clark, 2012). These miRNAs control various key cellular processes, such as proliferation, apoptosis, differentiation, and development, and are implicated in several human diseases, including cancer (Bhardwaj et al, 2010; Ryan et al, 2010). Earlier, it was demonstrated that $\sim 52.5 \%$ of miRNA genes are located in cancer-associated genomic regions, or in fragile sites, thus strongly suggested their role in cancer (Calin et al, 2004). Depending on the mRNA targets, miRNAs may function either as oncogenes (by inhibiting tumour suppressor genes) or as tumour suppressors (by inhibiting oncogenes) and thus have an important role in the progression and pathogenesis of cancer (Bhardwaj et al, 2010). According to recently published studies, a number of miRNAs have been shown to be differentially expressed in PC (Srivastava et al, 2014). These aberrantly expressed miRNAs have diverse pathological functions, such as regulating cell cycle progression, apoptosis, chemoresistance, angiogenesis, tumorigenicity, and metastatic potential (Srivastava et al, 2014). miR-345 was reported to be one of the top aberrantly expressed miRNAs in PC exhibiting significant downregulation (Bloomston et al, 2007; Lee et al, 2007; Szafranska et al, 2007); however, its role in PC pathogenesis has remained unexplored thus far.

In the current study, we demonstrate that miR-345 is significantly downregulated in PC, and its restoration in PC cells diminishes growth and induces apoptosis through activation of caspasedependent and -independent pathways. Moreover, we show that miR-345 directly targets the $3^{\prime}$ UTR of BCL2 and suppresses its expression at the protein level. Furthermore, we present evidence that the unopposed expression of BCL2 leads to abrogation of pro-apoptotic effects of miR-345. Together, these findings highlight the pro-apoptotic function of miR-345 in PC and support its utility as a candidate for diagnostic and therapeutic target.

\section{MATERIALS AND METHODS}

Cell culture and pancreatic tissue specimens. Human PC cell lines (MiaPaCa, Panc1, Colo-357, HPAF, ASPC-1, Panc10.05, Panc02.03, Panc03.27, BXPC3, CFPAC, CAPAN1, and SW1990) were maintained as monolayer cultures in DMEM or RPMI 1640 media (Invitrogen, Carlsbad, CA, USA) supplemented with 10\% fetal bovine serum (Atlanta Biologicals, Lawrenceville, GA, USA) and $100 \mu \mathrm{M}$ of penicillin and $100 \mu \mathrm{g} \mathrm{ml}^{-1}$ of streptomycin (Invitrogen). The hTERT-HPNE cells were maintained in Medium D containing 1 volume of M3 base (InCell Corp., San Antonio, TX, USA), 3 volumes of glucose-free DMEM, $5.5 \mathrm{~mm}$ glucose, $10 \mathrm{ng} \mathrm{ml}^{-1}$ epidermal growth factor, $50 \mu \mathrm{g} \mathrm{ml}^{-1}$ gentamicin (all from SigmaAldrich, St Louis, MO, USA), and 5\% fetal bovine serum. All the PC cell lines were cultured in humidified atmosphere at $37^{\circ} \mathrm{C}$ with $5 \%$ $\mathrm{CO}_{2}$ and media were replaced as needed. Cell lines were tested intermittently for mycoplasma contamination. Frozen pancreatic tissue (normal and malignant) specimens were obtained through the cooperative human tissue network at the University of Alabama at Birmingham under an institutional review board-approved protocol.

Plasmids and transfections. For the generation of stably miR345-overexpressing cell lines, MiaPaCa and Pancl cells were transfected with miR-345 expression (pCMV-miR-345) or control (pCMV-Neo) plasmid (Origene, Rockville, MD, USA) using $\mathrm{X}$-tremeGENE HP DNA transfection reagent (Roche, Indianapolis, IN, USA) as per the manufacturer's instructions. Stable populations of transfected cells were selected in media containing $200 \mu \mathrm{g} \mathrm{ml}^{-1}$ of G418 (Gibco, Grand Island, NY, USA), expanded and examined for stable miR-345 overexpression. For inhibition of miR-345 function, miR-345-overexpressing cells (Panc1-miR-345 and MiaPaCa-miR-345) were transiently transfected with antimiR-345 or miRNA inhibitor negative controls (Ambion, Austin, TX, USA) at $30 \mathrm{~nm}$ concentration using X-tremeGENE HP DNA transfection reagent. For BCL2 overexpression, cells were transiently transfected with pcDNA3-BCL2 construct (lacking 3'UTR region) generated by Stanley Korsmeyer Laboratory (procured through Addgene, Cambridge, MA, USA, plasmid \# 8768) or pcDNA3 (Invitrogen) as a control vector using X-tremeGENE HP DNA transfection reagent.

RNA isolation and real-time quantitative reverse-transcription PCR (qRT-PCR). Total RNA was extracted using TRIzol reagent as per the manufacturer's instruction (Invitrogen). Complementary DNA (cDNA) was synthesised using the high-capacity cDNA reverse transcription kit following manufacturer's instructions (Applied Biosystems, Carlsbad, CA, USA). Expression level of mature miR-345 was examined by following the strategy as described earlier (Srivastava et al, 2011). To examine the expression level of BCL2, real-time qRT-PCR was performed in 96-well plates using cDNA and SYBRGreen Master Mix (Roche) on an iCycler system (Bio-Rad, Hercules, CA, USA) with specific primers. Threshold cycle $(\mathrm{Ct})$ values for miR-345 and BCL2 were normalised against $\mathrm{Ct}$ values for U6 small nuclear RNA and GAPDH, respectively, and a relative fold change in expression was calculated. Details of the primers used in the study are provided in Supplementary Table 1 . The PCR conditions used were: cycle 1: $95^{\circ} \mathrm{C}$ for $10 \mathrm{~min}$, cycle $2(\mathrm{x} 40)$ : $95^{\circ} \mathrm{C}$ for $10 \mathrm{~s}$ and $58^{\circ} \mathrm{C}$ for $45 \mathrm{~s}$.

Cell growth assays. PC cells $\left(5 \times 10^{3}\right.$ cells per well $)$ were seeded in to 96 -well plates, and cultured up to 5 days. Media were replaced every day with fresh complete media. Cell growth was monitored each day using WST-1 assay kit (Roche Diagnostics, Mannheim, Germany) as described earlier (Bhardwaj et al, 2014; Deshmukh et al, 2015). Separately, miR-345-overexpressing cells were transiently transfected with BCL2 for $24 \mathrm{~h}$, cultured up to 5 days and growth monitored as described above.

Hoechst 33342 staining. Cells $\left(2.5 \times 10^{5}\right)$ were grown in glassbottom fluorodishes, washed with PBS, and incubated with $10 \mathrm{mg} \mathrm{ml}^{-1}$ Hoechst 33342 (Life Technologies, Grand Island, NY, USA) in the dark at room temperature for $10 \mathrm{~min}$. Cells were then observed and photographed using a Nikon Eclipse TE2000-U fluorescent microscope (Nikon Instruments Inc, Melville, NY, USA).

Apoptosis assay. Cells $\left(1 \times 10^{6}\right)$ were seeded in to six-well plates. After $24 \mathrm{~h}$, culture medium was replaced with fresh complete medium and cells were allowed to grow for next $48 \mathrm{~h}$. Subsequently, the extent of apoptosis was measured as previously described (Srivastava et al, 2012; Arora et al, 2015). In brief, cells were harvested and stained with 7-amino-actinomycin (7-AAD) and $\mathrm{PE}$ Annexin $\mathrm{V}$ using commercially available kit (BD Pharmingen, San Diego, CA, USA) followed by flow cytometry on a BD-FACS Canto II (Becton-Dickinson, San Jose, CA, USA). Percentage of the apoptotic cells was calculated using DIVA software version 6.1.3 (Becton-Dickinson).

Mitochondrial membrane potential $(\Delta \psi \mathrm{m})$ determination. Cells $\left(1 \times 10^{6}\right)$ grown in six-well plates were treated with $20 \mu \mathrm{g} \mathrm{ml}^{-1}$ of JC-1 dye (Life Technologies) for $20 \mathrm{~min}$ and incubated at $37^{\circ} \mathrm{C}$ in the dark. Following incubation, cells were harvested by 
trypsinising, washed, resuspended in $500 \mu \mathrm{l}$ of PBS, and analysed by flow-cytometry on a BD-FACS Canto II. Percentage of loss in $\Delta \psi \mathrm{m}$ was calculated using DIVA software version 6.1.3.

Subcellular fractionation. The cytoplasmic, mitochondrial, and nuclear fractions from low- and high-miR-345-expressing PC cells were prepared using commercially available kit as per the manufacture's protocol (Mitosciences, Oregon, OR, USA).

Western blot analysis. Protein isolation and western blotting were performed as described earlier (Srivastava et al, 2011; Tyagi et al, 2014). Immunodetection was carried out using specific antibodies ( $1: 1000)$ against BCL2, cytochrome $c$, cleaved caspase-3, cleaved caspase-7, PARP-1, apoptosis-inducing factor (AIF; rabbit monoclonal), HDAC (mouse monoclonal; Cell Signalling, Danvers, MA, USA), $\alpha$-tubulin (rabbit monoclonal), and VDAC (goat monoclonal; Santa Cruz Biotechnology, Dallas, TX, USA). $\beta$-Actin (mouse monoclonal; 1:20000; Sigma-Aldrich, St Louis, MO, USA), $\alpha$-tubulin, VDAC, and HDAC served as an control for total, cytoplasmic, mitochondrial, and nuclear fractions, respectively. All secondary antibodies (Santa Cruz Biotechnology) were used at 1:2500 dilution. Proteins were visualised with the SuperSignal West Femto Maximum sensitivity substrate kit (Thermo Scientific, Logan, UT, USA) and the signal detected using an LAS-3000 image analyzer (Fuji Photo Film Co., Tokyo, Japan).

Caspase-3/7 activity assay. Caspase-3/7 activity was determined using a commercially available Caspase-Glo $3 / 7$ assay kit (Promega, Madison, WI, USA) as per the manufacturer's protocol. Briefly, cells were cultured in 96-well plate for $48 \mathrm{~h}$ and $100 \mu \mathrm{l}$ of Caspase-Glo 3/7 reagent was added. Subsequently, cells were incubated at room temperature for $1 \mathrm{~h}$ and luminescence measured using VICTOR-X3 Multilabel Plate Reader (Perkin Elmer, Santa Clara, CA, USA).

Dual luciferase $3^{\prime}$ UTR-reporter assay. For the validation of BCL2 as a direct target of miR-345, cells were transiently co-transfected for $24 \mathrm{~h}$ with $200 \mathrm{ng}$ of pLuc3U-BCL2 target-reporter plasmid containing BCL2 3 'UTR region (Signosis, Santa Clara, CA, USA) along with $0.25 \mu \mathrm{g}$ of control reporter plasmid (pRL-TK; Promega; plasmid containing a Renilla reniformis luciferase gene downstream of the thymidine kinase (TK) promoter. Moreover, as a control, we also generated a mutant BCL2 $3^{\prime}$ UTR (MUT-BCL2 $3^{\prime}$ UTR) reporter construct by site-directed mutagenesis in the putative target region of miR-345 using Quickchange XL sitedirected mutagenesis kit (Agilent Technologies, Santa Clara, CA, USA) and transiently transfected as described above. After $48 \mathrm{~h}$ of transfection, cells were harvested in reporter lysis buffer (Promega). Firefly and Renilla luciferase activities were measured using a dual-luciferase assay kit (Promega) according to the manufacturer's instructions. The data are represented as the ratio of firefly to Renilla luciferase activity.

Statistical analysis. All the experiments were performed at least three times and numerical data expressed as mean \pm s.d. The expression profiles of miR-345 in malignant pancreatic versus normal tissues were analysed using unpaired one-tailed Student's $t$-test. A pairwise comparison was performed to check if miR-345 had lower expression level in PCs than in NPs. A value of $P<0.05$ was considered to be significant throughout the study.

\section{RESULTS}

miR-345 is downregulated in PC. We examined the expression of miR-345 in a set of normal $(n=7)$ and cancerous $(n=21)$ pancreatic tissues, in an in vitro progression model cell lines (hTERT-HPNE and derived lines; Campbell et al, 2008) and a panel of established PC cell lines. Our data reveal that miR-345 is downregulated in the majority of PC cases as compared with the normal pancreas. Relative mean expression of miR-345 in the normal pancreatic tissues is significantly higher (41.93, $P$-value $\leqslant 0.001)$ than that of the malignant pancreatic tissues (11.85; Figure 1A). Furthermore, we observe that the expression of miR-345 is lost gradually in PC progression model cell lines (Figure 1B); whereas a differential expression pattern is seen among various established PC cell lines (Figure 1C). Notably, expression of miR-345 is relatively downregulated in poorly differentiated cell lines as compared with well-differentiated cell lines (Figure 1C). Altogether, our data demonstrate the downregulated expression of miR-345 in PC.

miR-345 promotes apoptosis in PC cells. To investigate the biological relevance of miR-345 downregulation in PC, we stably overexpressed it in two poorly differentiated PC cell lines, Panc1 and MiaPaCa (exhibiting low endogenous expression of miR-345). miR-345 overexpression was confirmed by real-time RT-PCR analysis. The data show that Panc1-miR-345 and MiaPaCa-miR345 cells have $\sim 63$ - and $\sim 51$-fold overexpression of miR-345, respectively, as compared with the control cell lines (Supplementary Figure 1). Growth analyses of miR-345-transfected cells revealed $\sim 57.8 \%$ and $\sim 52.6 \%$ reduction in overall growth by 5th day in Panc1-miR-345 and MiaPaCa-miR-345, respectively, relative to control vector (Panc1-Neo and MiaPaCa-Neo)-transfected cell lines (Figure 2A). Morphological examination demonstrated that miR-345-overexpressing PC cells became round and shrunken, and many of them detached from the bottom of the culture plate (data not shown). Therefore, we next examined the effect of miR-345 overexpression on chromatin condensation and nuclear fragmentation by Hoechst 33342 staining to confirm if the reduced growth and morphological alterations resulted from cellular apoptosis. Data demonstrate the presence of cells with fragmented nuclei and condensed chromatin in miR-345-overexpressing PC cells (Figure 2B), suggesting apoptosis induction upon restoration of miR-345 in PC cells. We then confirmed apoptosis induction by performing Annexin $\mathrm{V}$ and 7-AAD staining followed by flow cytometry. The data reveal a higher apoptotic index (Annexin V-positive/7-AAD-negative cells) in Panc1-miR345 (48.5\%) and MiaPaCa-miR-345 (36.5\%) cells, as compared with their respective control cells, that is, Panc1-Neo (21.2\%) and MiaPaCa-Neo (17\%; Figure 2C). Importantly, the effect of miR-345 restoration on apoptosis was abolished following treatment of miR345-overexpressing PC cells with anti-miR-345 (Figure 2C). Taken together, these results indicate that miR-345 promotes apoptosis in PC cells.

miR-345 activates mitochondrial pathways of apoptosis in PC. Mitochondria are known to have a central role in apoptosis (Elkholi et al, 2014; Bhat et al, 2015). Therefore, we examined the effect of miR-345 on the mitochondrial membrane potential $(\Delta \psi \mathrm{m})$, and localisation/activation of the key proteins involved in apoptosis. Our flow cytometry analysis revealed a high proportion (35.6\% and $29.2 \%$, respectively) of damaged mitochondria/loss of $\Delta \psi \mathrm{m}$ in miR-345-overexpressing Pancl and MiaPaCa cells as compared with their respective controls (Figure 3A). Moreover, we observed that the loss of $\Delta \psi \mathrm{m}$ was associated with increased levels of cytochrome $c$ in the cytosol with a concomitant decrease in the mitochondria of miR-345-overexpressing cells (Figure 3B). Similarly, we also observed increased levels and activity of effector caspases (cleaved caspases-3 and -7) (Figure 3C and Supplementary Figure 2) along with PARP-1 cleavage in miR345-overexpressing PC cells (Figure 3C). Interestingly, the effects of miR-345 overexpression on $\Delta \psi \mathrm{m}$, cytochrome $c$ translocation, and activation of caspases were attenuated by treatment with miR345 inhibitor (Figure 3A-C). To explore the possibility of caspaseindependent apoptosis, we examined the levels of AIF, known to induce apoptosis in a caspase-independent manner (Cande et al, 

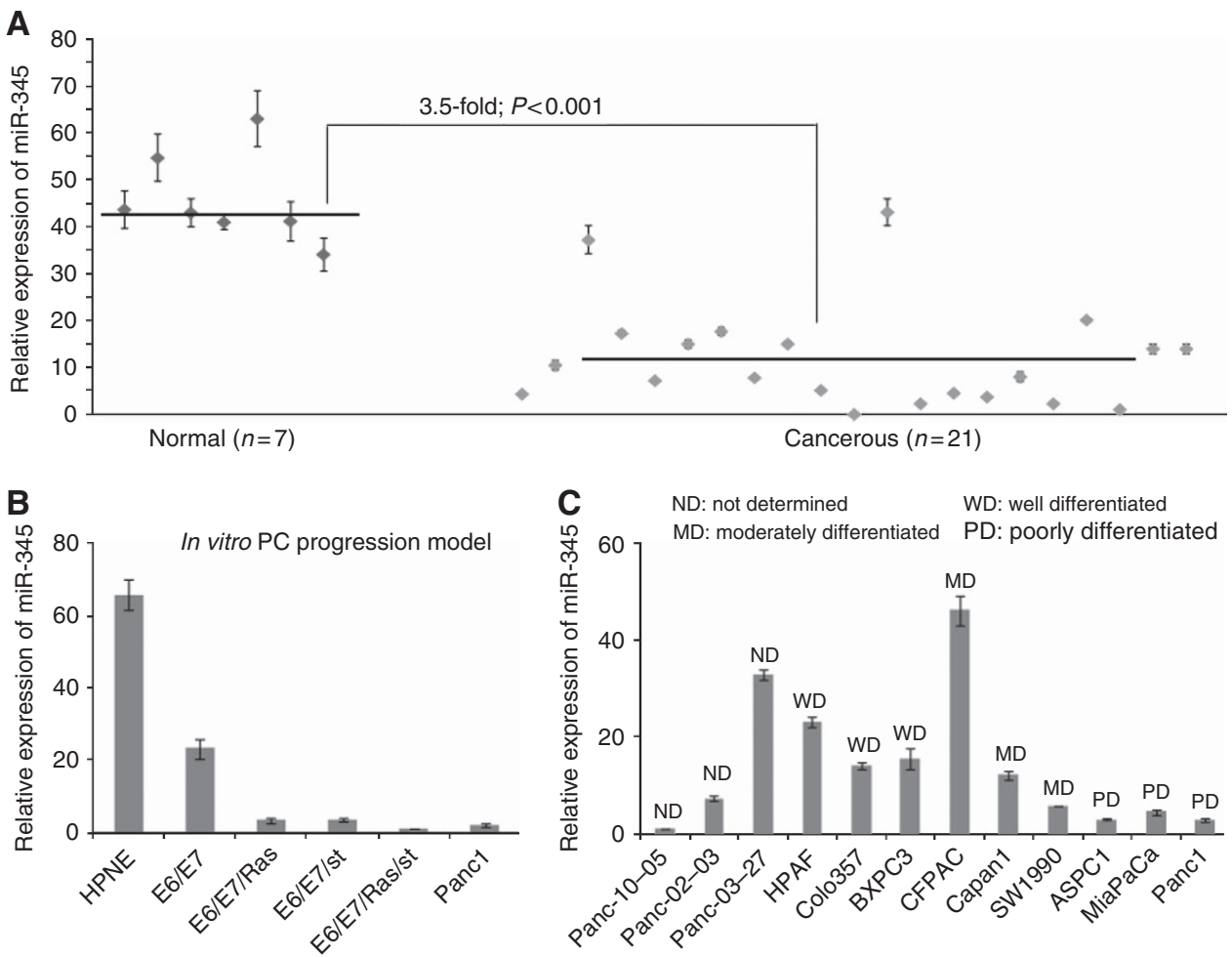

Figure 1. miR-345 is downregulated in pancreatic cancer. (A) miR-345 expression profiling was performed in normal $(n=7)$ and cancerous $(n=21)$ pancreatic tissue specimens. A decreased expression of miR-345 is observed in majority of cancer tissues as compared with the normal pancreas. Dark lines indicate mean expression level of miR-345 of the group and the fold reflects the ratio between the mean values of miR-345 expression in normal vs cancer cases. (B) Expression analysis of miR-345 in hTERT-HPNE PC in vitro progression model demonstrates a gradual decrease in the expression of miR-345. (C) A differential expression pattern of miR-345 is observed in PC cell lines. All the poorly differentiated cell lines exhibit relatively lower expression as compared with the well-differentiated cell lines. U6 small nuclear RNA served as an internal control. A relative quantity of miR-345 was determined using the $2^{-\Delta \Delta C T}$ method. Bars represent the mean \pm s.d. $(n=3)$.

2004). The data reveal that the level of nuclear AIF is increased with a concomitant decrease in its level in the mitochondrial fraction in miR-345-overexpressing Pancl and MiaPaCa cells (Figure 3D). Furthermore, we observe that the level of mitochondrial AIF is regained in miR-345-overexpressing cells when they are treated with anti-miR-345 (Figure 3D). Together, our findings suggest that miR-345 induces apoptosis in PC cells by promoting caspase-dependent as well as -independent pathways of apoptosis.

miR-345 negatively regulates $B C L 2$ through direct binding to its $\mathbf{3}^{\prime}$ UTR. To identify the target of miR-345, we performed in silico analysis using the algorithms Target Scan (http://www.targetscan.org) and miRanda (http://www.microrna.org), and identified BCL2, an important molecule of the anti-apoptotic gene family, as putative target of miR-345. Further investigation revealed the presence of a 8-merbinding site for miR-345 in the $3^{\prime} \mathrm{UTR}$ of the BCL2 transcript (Figure 4A). To validate the potential targeting of BCL2 by miR-345, we examined its expression in a miR-345-overexpressing Pancl and $\mathrm{MiaPaCa}$ cells. Our investigation revealed no change in the expression of $B C L 2$ at the transcript level (Figure 4B; upper panel); however, its expression decreased at the protein level in both Panc1-miR-345 and MiaPaCa-miR-345 cells as compared with their respective control cells (Figure 4B; upper panel), thus suggesting its translational repression by miR-345. To test whether BCL2 is a direct target of miR-345, control and miR-345-overexpressing PC cells were transiently transfected with a luciferase reporter plasmid containing a region of BCL2 $3^{\prime} \mathrm{UTR}$ having a wild-type or mutated miR-345 target site (Figure $4 \mathrm{C}$ ). As shown in Figure 4D, our data demonstrate that miR-345 significantly suppressed the luciferase activity of the reporter plasmid with wildtype-BCL2 3'UTR in Panc1-miR-345 and MiaPaCa-miR-345 ( 69\% and $\sim 83 \%$, respectively) as compared with that in control cells.
Furthermore, cells transfected with mutated-3'UTR did not show any response to the suppressor activity of miR-345 (Figure 4D). Altogether, our data suggest that BCL2 is a direct target of miR-345.

BCL2 is involved in the miR-345-mediated activation of apoptotic pathways in PC cells. Following identification of $B C L 2$ as a direct target of miR-345, we further examined its significance in miR-345-mediated induction of apoptosis of PC cells. For this, expression vector of BCL2, which encodes the entire coding sequence of BCL2, but lacks the $3^{\prime} \mathrm{UTR}$, was transiently transfected into the miR-345-overexpressing PC cells (Panc1-miR345 and MiaPaCa-miR-345), and the effects on proteins associated with apoptosis pathways were analysed. Our immunoblot analysis shows that forced expression of BCL2 efficiently blocked the miR345 -induced activation of caspases, cleavage of PARP-1, and prevents the nuclear translocation of AIF (Figure 5A). Furthermore, we also examined the effect of BCL2 overexpression on the miR-345 decreased growth of PC cells. Our data demonstrate that forced expression of BCL2 abrogated the growth inhibitory effect of miR-345 in both Pancl and MiaPaCa cells (Figure 5B). Together, these data suggest that miR-345 promotes apoptosis by suppressing the expression of BCL2, which triggers the mitochondrial pathway of apoptosis (Figure 6).

\section{DISCUSSION}

The recent discovery of miRNAs has revealed a novel mechanism of gene regulation and provided new avenues for cancer research (Srivastava et al, 2014). These miRNAs are highly deregulated in various malignancies, including PC, and their aberrant expression 


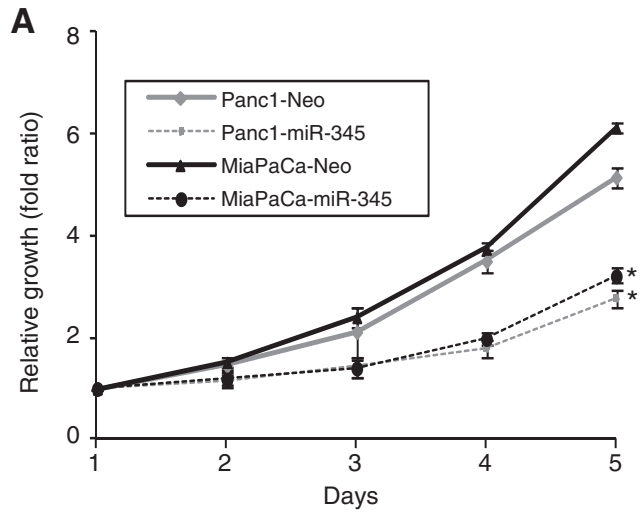

C
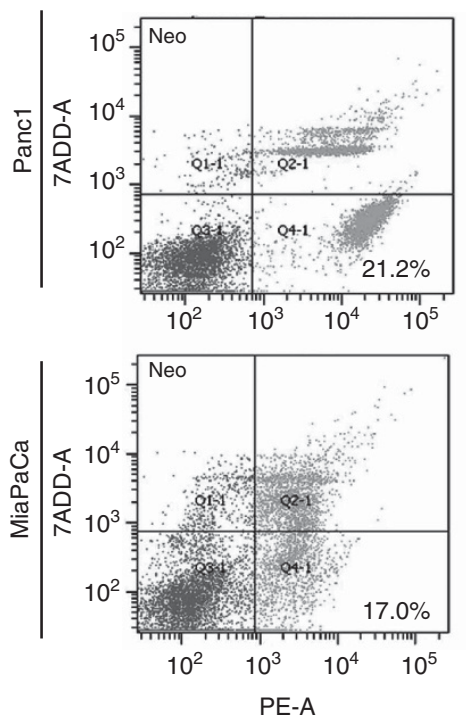

B
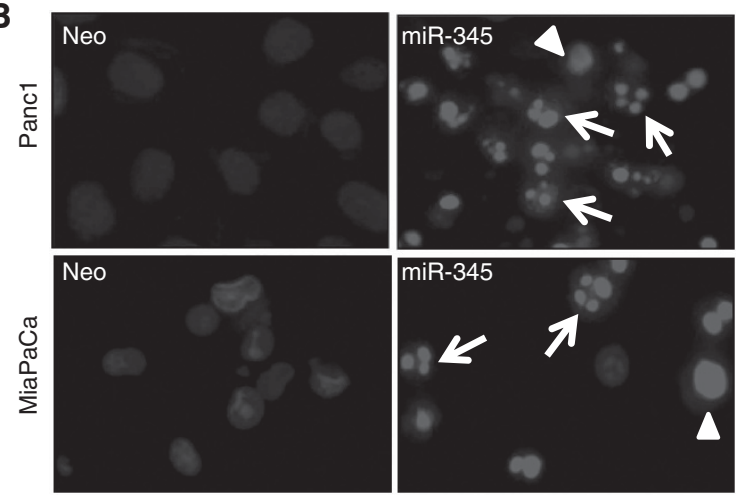

miR-345
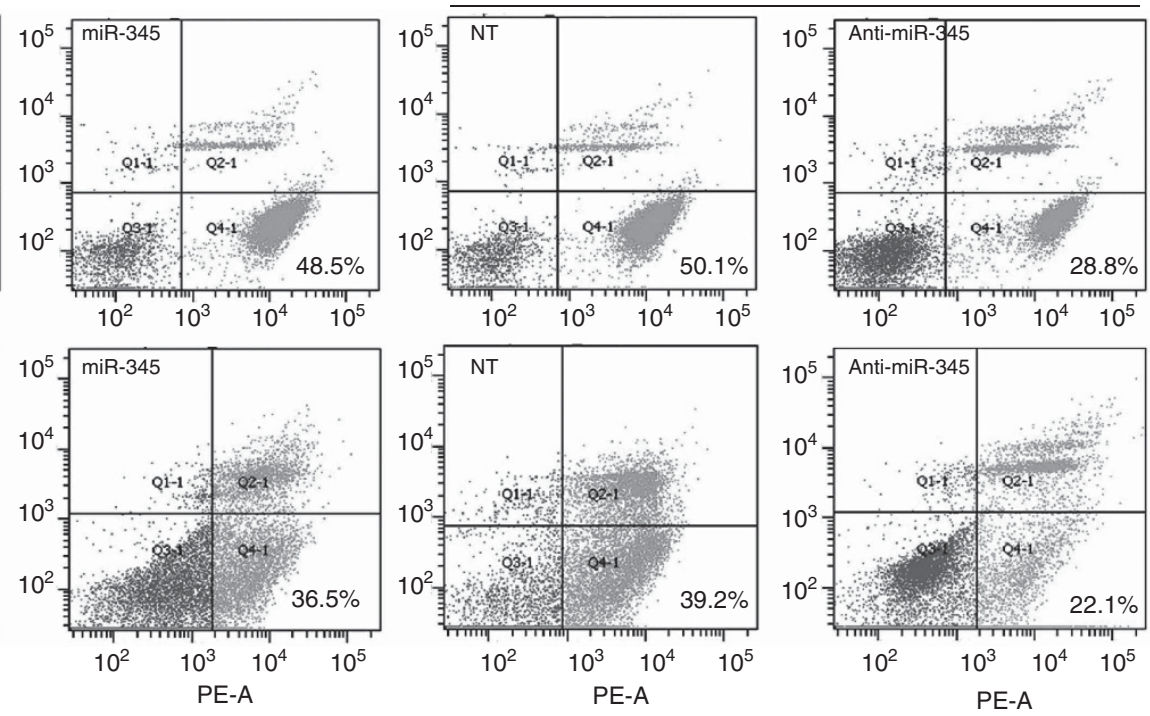

Figure 2. miR-345 restoration induces apoptosis in PC cells. (A) Cell growth was monitored by WST-1 assay daily up to 5 days. Data are presented as fold change in comparison to the cell growth on day 1 . Data (mean \pm s.d., $n=3 ;{ }^{*} P<0.05$ ) show decreased growth of miR-345-overexpressing PC cells relative to their respective controls. (B) PC cells were cultured up to $48 \mathrm{~h}$ in glass bottom fluorodishes, stained with Hoechst 33342 , and examined and photographed using a fluorescent microscope. miR-345-overexpressing PC cells show apoptotic characteristics as evident by condensed (arrowhead) and fragmented (arrow) nuclei. Micrographs represent random fields of view (magnification $\times 400$ ). (C) miR-345-overexpressing PC cells along with their control cells were seeded in six-well plate and media replaced after $24 \mathrm{~h}$. After next $48 \mathrm{~h}$ incubation, cells were washed, stained with PE Annexin V, and analysed for apoptosis by flow cytometry. In parallel, miR-345-overexpressing PC cells were transiently transfected with anti-miR-345 or non-target (NT) sequence control, cultured up to $48 \mathrm{~h}$ and processed for apoptosis analysis. Histograms are representative of three independent experiments (Panc1, upper panel; MiaPaCa, lower panel). Data suggest a pro-apoptotic role of miR-345 in PC cells.

has been linked with initiation, progression, and dissemination of cancer cells. miR-345, which was identified to be one of the most significantly downregulated miRNA in PC (Bloomston et al, 2007; Lee et al, 2007; Szafranska et al, 2007), was studied for its functional role in PC. In corroboration with published reports, we also observed significant downregulation of miR-345 in majority of pancreatic tumour tissues and established PC cell lines. Moreover, we also observed a progressive loss of miR-345 in an in vitro progression model of PC. Although the mechanism(s) underlying miR-345 downregulation in PC is yet to be determined, it is likely that both genetic and non-genetic mechanisms are involved. An earlier study by Loukopoulos and co-workers demonstrated that chromosomal region $14 \mathrm{q} 32-33$, which harbours the gene encoding miR-345, is frequently lost in PC (Loukopoulos et al, 2007). Low expression of miR-345 has been observed in other malignancies as well and associated with clinicopathological features. miR-345 downregulation correlated with lymph node metastasis in clinical specimens of colorectal cancer, and its restoration suppressed colon cancer cell proliferation and invasiveness (Tang et al, 2011).
Moreover, Pogribny et al (2010) identified a role of miR-345 downregulation in the development of cisplatin resistance in breast cancer cells. These studies strongly suggest that downregulation of miR-345 might be an important event in PC initiation and/or progression at least in a significant number of cases.

Apoptosis is a tightly regulated cell death process that has a critical role in growth and tissue homeostasis. In fact, the inherent ability to resist apoptosis is a hallmark of tumorigenesis. Moreover, preclinical studies in PC have shown that inhibition of apoptosis yields favorable response to gemcitabine cytotoxicity (Bauer et al, 2015; Guo et al, 2015). Therefore, enormous research has been done to understand the mechanisms of apoptosis resistance, associated regulatory pathways, and molecular targets for the development of novel anti-cancer therapies. In this regard, our study identified miR-345 downregulation as an important mechanism that confers apoptosis resistance to PC cells through mitochondrial pathways. Mitochondria have been very well recognised for their central role in regulating apoptosis. It is considered that loss of the mitochondrial membrane potential 
A
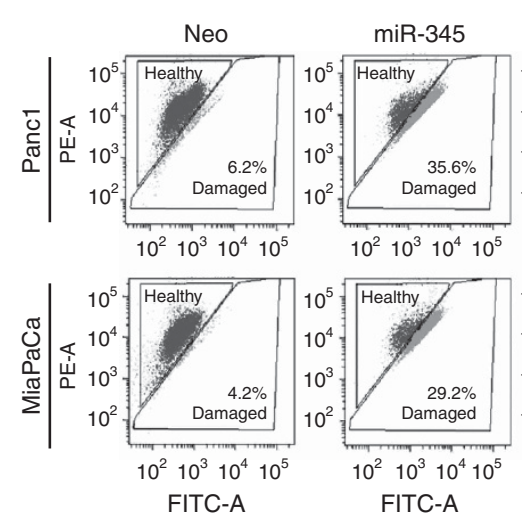

B

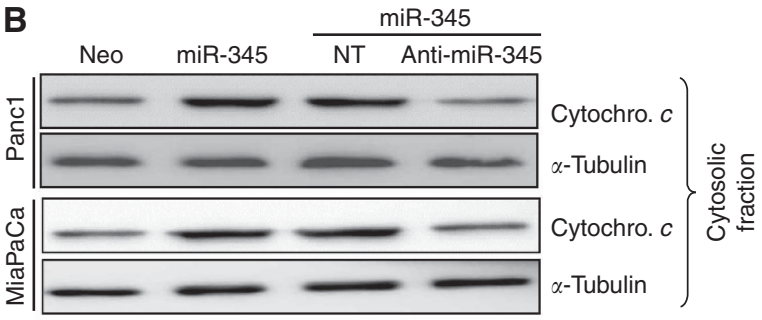

miR-345

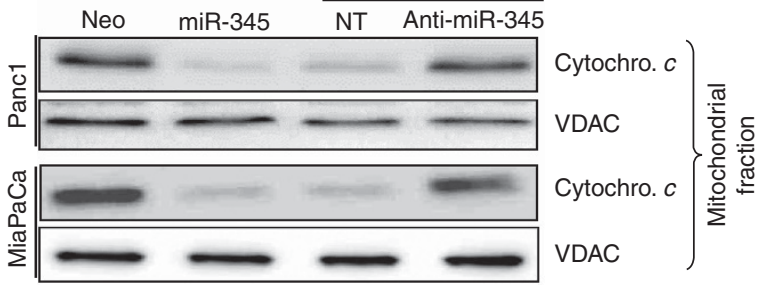

miR-345 Anti-miR-345
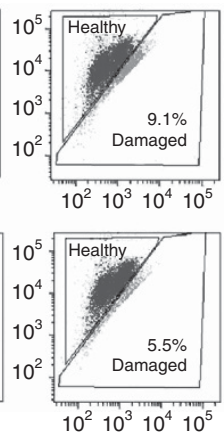

FITC-A
C

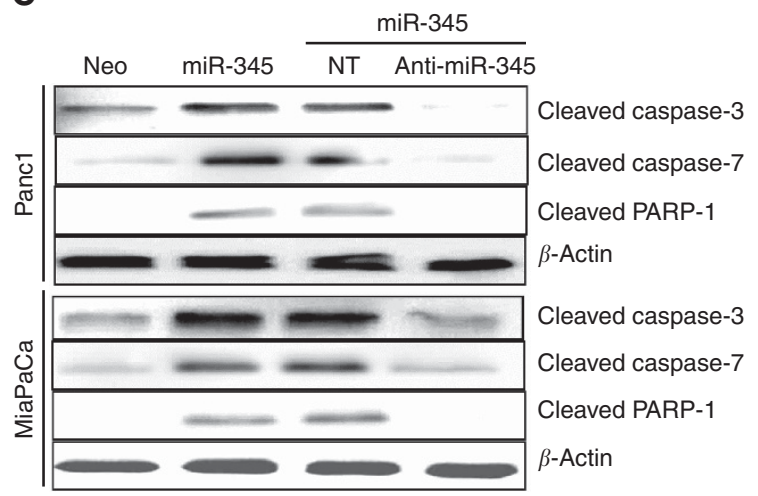

D

miR-345

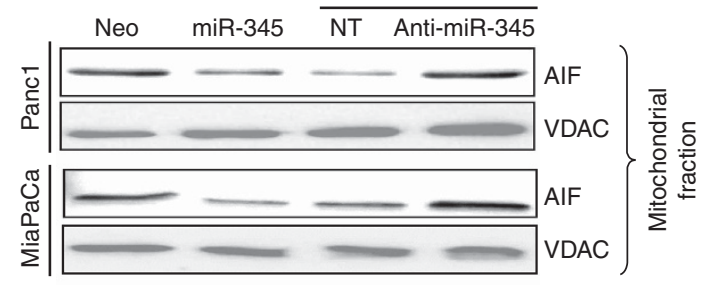

miR-345

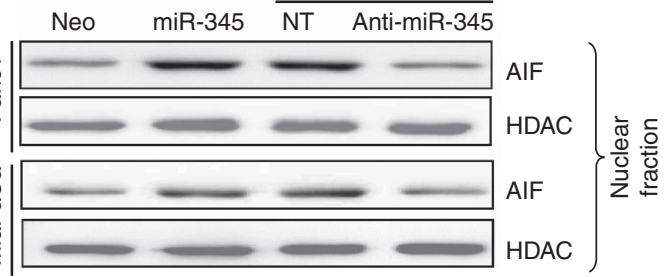

Figure 3. miR-345 promotes apoptosis through mitochondrial pathways. (A) miR-345-overexpressing PC (Panc1-miR-345 and MiaPaCa-miR-345) cells were transiently transfected with anti-miR-345 or non-target (NT) sequence control. After $48 \mathrm{~h}$ of transfection, cells were stained with JC-1 as described in Materials and Methods and subjected to flow cytometry. Dot plot shows cell population with intact mitochondria (having high $\Delta \psi$ m; in blue); and population with damaged mitochondria (having low $\Delta \psi \mathrm{m}$; in orange). Results demonstrate a loss of $\Delta \psi \mathrm{m}$ in miR-345-overexpressing PC cells, an effect that was abrogated upon treatment with anti-miR-345. (B-D) Total, cytoplasmic, nuclear, or mitochondrial protein fractions were collected from low- and high-miR-345-expressing PC cells; and high-miR-345-expressing cells transiently transfected with anti-miR-345 or NT sequence control. Thereafter, expression of (B) cytochrome $c$ (in cytosolic and mitochondrial fractions), (C) cleaved caspase-3, caspase-7, and PARP-1 (in total fraction) and (D) AIF (in nuclear and mitochondrial fractions) was examined by immunoblot assay. $\beta$-Actin, $\alpha$-tubulin, VDAC, and HDAC were used as loading control for the total, cytosolic, mitochondrial, and nuclear fractions, respectively.

$(\Delta \Psi \mathrm{m})$ is the important event for the mitochondria-mediated apoptosis pathways (Gupta et al, 2009). Following the collapse of $\Delta \Psi \mathrm{m}$, cytochrome $c$ is released into the cytosol, where it forms complex with apoptotic protease-activating factor-1 and ATP. This complex then binds to pro-caspase-9, and causes its cleavage, which further initiates the activation of effector caspase- 3 and -7. These effector caspases then cleave PARP-1, a known cellular substrates of caspases, whose cleavage by caspases is considered to be a characteristic of caspase-dependent apoptosis (Elmore, 2007). Along these lines, we demonstrated a disruption of $\Delta \Psi \mathrm{m}$ in PC cells following miR-345 overexpression, release of cytochrome $c$ from mitochondria to cytosol, and further activation of effectors caspase-3, caspase-7, and subsequent PARP-1 cleavage. Although caspase activation is considered a hallmark of apoptotic cell death, mitochondrially mediated apoptosis induction also occurs in a caspase-independent manner (Borner and Monney, 1999; Sperandio et al, 2000). AIF is localised to the mitochondrial intermembrane space, and upon apoptotic stimuli, AIF translocates to the nucleus where it causes chromatin condensation and nuclear fragmentation, resulting in cell death (Cande et al, 2004). Interestingly, in our study, we found that miR-345 overexpression induced the translocation of AIF to the nucleus. Thus, miR-345induced apoptosis involved both cytochrome $c$ (caspase-dependent) and AIF (caspase-independent) in the mitochondrial apoptotic pathway.

Findings from our study are quite interesting as we identified anti-apoptotic BCL2 to be a direct target of miR-345. Our study suggested that miR-345 downregulation-mediated upregulation of BCL2 could be a key event in triggering apoptosis resistance in PC cells. Several lines of evidence show that BCL-2 is overexpressed in variety of malignancies, including PC (Bold et al, 2001; Sun et al, 2002; Scherr et al, 2014), and its elevated levels have been shown to be associated with apoptosis-resistance and enhanced metastatic potential in PC (Bold et al, 1999, 2001). BCL2 keeps a check on the release of cytochrome $c$ and AIF from mitochondria by preventing the disruption of mitochondrial membrane potential (Susin et al, 1996; Harris and Thompson, 2000; Tait and Green, 2010). We demonstrated that exogenous expression of BCL2 abrogated the miR-345-induced activation of caspases and prevented the translocation of AIF to the nucleus, favouring cell survival. Yang et al (1997) in their study investigated that overexpression of BCL2 prevents the initiation of the cellular apoptotic programme by 
A

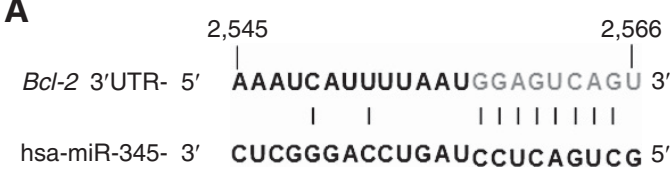

B

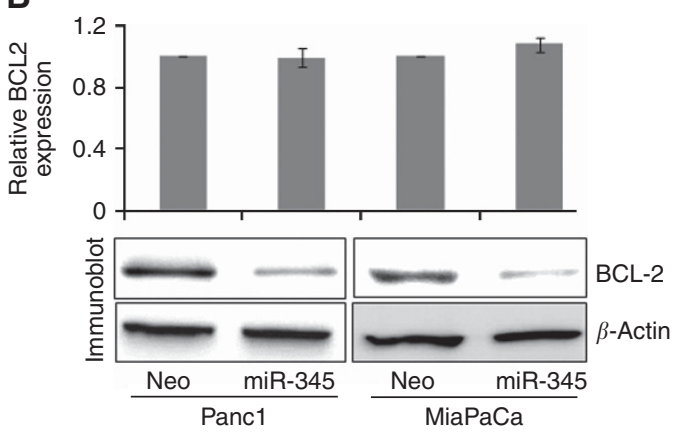

C $2,552 \quad 2,566$

WT 3'UTR- 5' Uं U U A A U G G A G U C A G Ù $3^{\prime}$

| | | | | | | |

miR-345- 3

C CUCAGUCG 5

$2,552 \quad 2,566$

MUT $3^{\prime}$ UTR-5' U' U U A A U G G C A C C A G U' $3^{\prime}$

। । । । ।

miR-345- $3^{\prime}$

C CUCAGUCG $5^{\prime}$

D

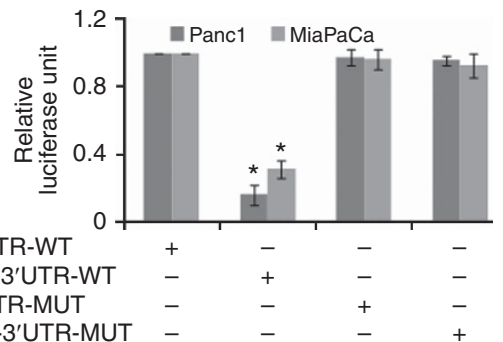

Figure 4. miR-345 suppresses BCL2 expression in PC cells through directly targeting its $3^{\prime}$ UTR. (A) In silico analysis (using algorithms of Target Scan and miRanda) showing miR-345-binding sites in BCL2 3'UTR. (B) Total RNA and protein from control and miR-345-overexpressing PC cells were isolated and expression of BCL2 was examined by qRT-PCR and immunoblot analysis. GAPDH and $\beta$-actin served as internal controls for RTPCR and immunoblot analysis, respectively. Data show that expression of BCL2 remained unaltered at the transcript level, but was repressed at the protein level in both Panc1-miR-345 and MiaPaCa-miR-345 cells. (C) Schematic representation of BCL2 3'UTR with either wild-type (WT) or mutant (MUT) miR-345 target site. MUT-3'UTR construct carries three nucleotides variation in the seed matching region of the target site to disrupt binding of miR-345. (D) PC cells were grown in six-well plate and transiently co-transfected with the luciferase promoter-reporter plasmids containing WT or MUT BCL2-3'UTR along with pRL-TK construct (transfection efficiency control) for $24 \mathrm{~h}$. Thereafter, total protein was collected in passive lysis buffer and subsequently firefly/Renilla luciferase activities were measured using a dual-luciferase assay system. Data (mean $\pm \mathrm{s}$.d.; $n=3$ ) are presented as fold change in normalised luciferase activity. ${ }^{\star} P<0.05$. Results show that miR-345 suppresses the luciferase activity of the reporter plasmid containing WT 3'UTR of BCL2.

A

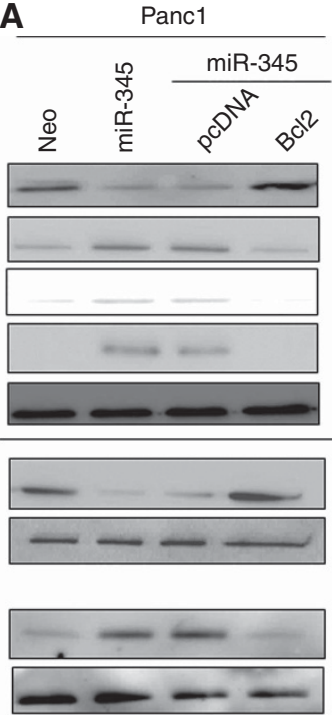

$\mathrm{MiaPaCa}$

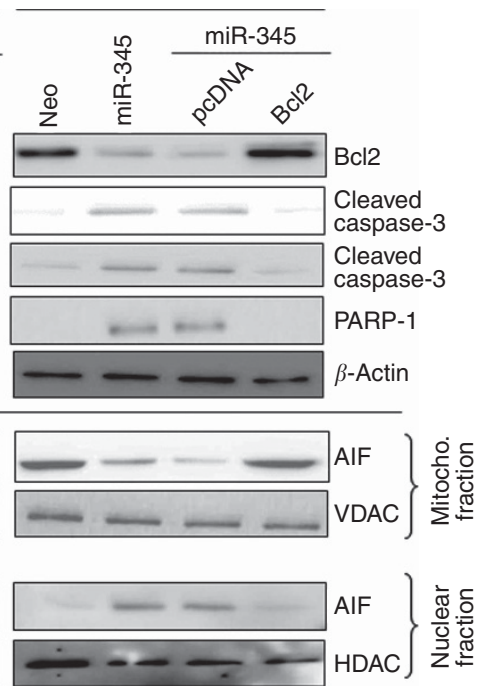

B
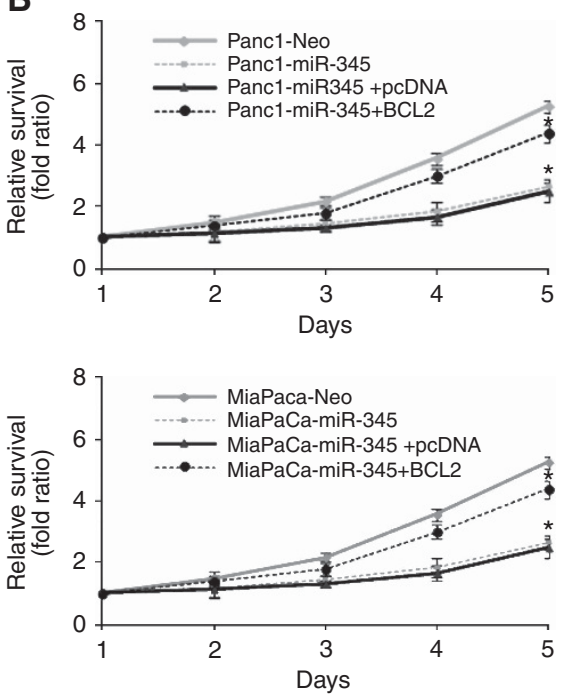

Figure 5. miR-345-mediated downregulation of BCL2 activates apoptosis. (A) miR-345-overexpressing PC cells were transiently transfected with BCL2-overexpressing (pcDNA3-BCL2) or control ( $\mathrm{PCDNA3}$ ) plasmids. After $48 \mathrm{~h}$ of transfection, total, nuclear, and mitochondrial fractions were collected and effect on the expression of BCL2, cleaved caspases-3 and -7, and cleaved PARP-1 (in total) and AIF (in nuclear and mitochondrial) was examined by immunoblot analysis using specific antibodies. $\beta$-Actin, VDAC, and HDAC were used as loading control for the total, mitochondrial, and nuclear fractions, respectively. Data demonstrate that forced expression of BCL2 blocked the miR-345-induced activation of caspases, PARP-1, and prevents the nuclear translocation of AIF. (B) Panc1-miR-345 and MiaPaCa-miR-345 cells were transiently transfected with BCL2-overexpressing or control plasmids. After $24 \mathrm{~h}$ of transfection, cells were trypsinised, counted, and seeded (5 $\times 10^{3}$ per well) in 96 -well plate. Thereafter, cell growth was monitored by WST-1 assay daily upto 5 days. Data (mean \pm s.d.; $n=3$ ) presented as fold change as compared with the cell growth on day 1. ${ }^{*} P<0.05$. Results show that forced expression of BCL2 abrogated the growth inhibitory effect of miR-345 in PC cells.

blocking the release of cytochrome $c$ from mitochondria. Moreover, a separate study by Susin et al (1998) demonstrated that BCL2 interferes with permeability transition pores and prevents the release of AIF. In this regard, the ability of miR-345 to downregulate the anti-apoptotic gene BCL2 is highly significant. 


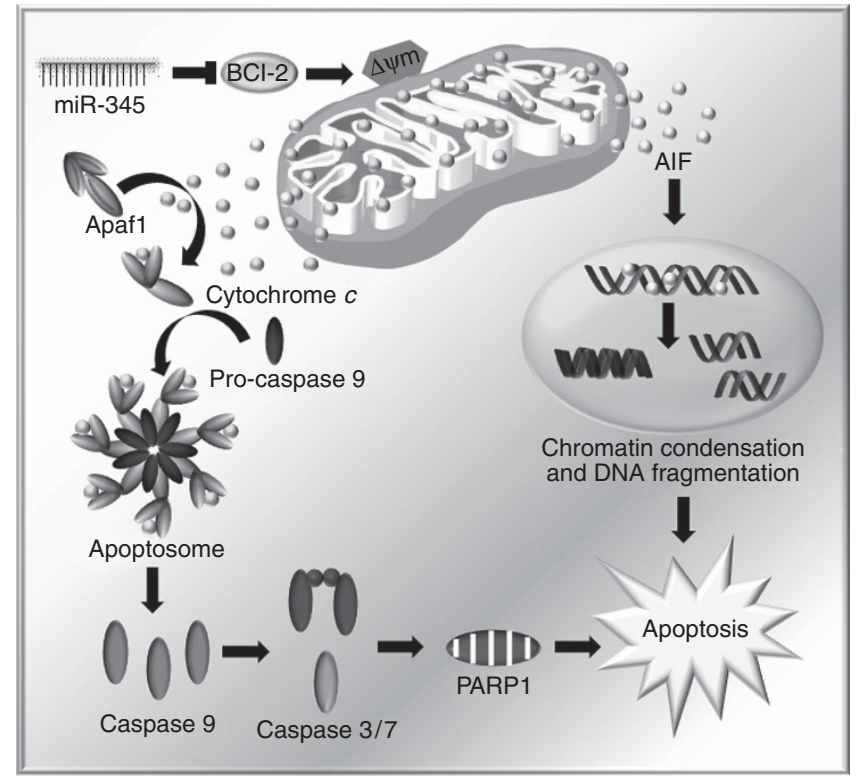

Figure 6. The proposed mechanistic model of miR-345-induced apoptosis in PC cells. The anti-apoptotic BCL2 protein guards the outer mitochondrial membrane and controls apoptosis by maintaining the mitochondrial permeability. miR-345 inhibits BCL2 through its direct binding, and its inhibition result in the loss of $\Delta \Psi \mathrm{m}$, which allows cytochrome $c$ to be released from the mitochondria into the cytosol. Released cytochrome $c$ then binds with apoptotic protease-activating factor-1 and procaspase-9, forms apoptosome, activates caspases-9 and $-3 / 7$ and PARP1 cleavage, and causing apoptosis. At the same time of cytochrome $c$ release, AIF is also liberated from the mitochondria, translocates to the cell nucleus, and causes DNA fragmentation and condensation resulting in cell death.

In summary, we showed that downregulation of miR-345 was a frequent event in PC, and this downregulation significantly correlated with PC progression. Furthermore, ectopic expression of miR-345 in PC cells dramatically reduced cell growth and induced apoptosis. We further identified BCL2 as functional target of miR-345, and proved the involvement of the mitochondrial pathway in miR-345-promoted apoptosis. Our findings thus suggest an essential role of miR-345 in regulating apoptosis, and implicate the potential application of miR-345 in PC therapy.

\section{ACKNOWLEDGEMENTS}

We thank Dr. M Ouellette, UNMC for kindly providing the hTERT-HPNE in vitro progression model of pancreatic cancer as a gift. This work was supported by funding from NIH/NCI (CA167137, CA175772, CA185490 (to APS) and CA169829, CA186233 (to SS)) and USAMCI.

\section{CONFLICT OF INTEREST}

The authors declare no conflict of interest.

\section{REFERENCES}

Arora S, Bhardwaj A, Singh S, Srivastava SK, McClellan S, Nirodi CS, Piazza GA, Grizzle WE, Owen LB, Singh AP (2013) An undesired effect of chemotherapy: gemcitabine promotes pancreatic cancer cell invasiveness through reactive oxygen species-dependent, nuclear factor kappaB- and hypoxia-inducible factor 1alpha-mediated up-regulation of CXCR4. J Biol Chem. 288(29): 21197-21207.

Arora S, Tyagi N, Bhardwaj A, Rusu L, Palanki R, Vig K, Singh SR, Singh AP, Palanki S, Miller ME, Carter JE, Singh S (2015) Silver nanoparticles protect human keratinocytes against UVB radiation-induced DNA damage and apoptosis: potential for prevention of skin carcinogenesis. Nanomedicine 11(5): 1265-1275.

Bauer C, Hees C, Sterzik A, Bauernfeind F, Mak'Anyengo R, Duewell P, Lehr HA, Noessner E, Wank R, Trauzold A, Endres S, Dauer M, Schnurr M (2015) Proapoptotic and Antiapoptotic Proteins of the Bcl-2 Family Regulate Sensitivity of Pancreatic Cancer Cells Toward Gemcitabine and T-Cellmediated Cytotoxicity. J Immunother 38(3): 116-126.

Bhardwaj A, Singh S, Singh AP (2010) MicroRNA-based cancer therapeutics: big hope from small RNAs. Mol Cell Pharmacol 2(5): 213-219.

Bhardwaj A, Srivastava SK, Singh S, Arora S, Tyagi N, Andrews J, McClellan S, Carter JE, Singh AP (2014) CXCL12/CXCR4 signaling counteracts docetaxel-induced microtubule stabilization via p21-activated kinase 4-dependent activation of LIM domain kinase 1. Oncotarget 5(22): $11490-11500$.

Bhat TA, Kumar S, Chaudhary AK, Yadav N, Chandra D (2015) Restoration of mitochondria function as a target for cancer therapy. Drug Discov Today 20(5): 635-643.

Bloomston M, Frankel WL, Petrocca F, Volinia S, Alder H, Hagan JP, Liu CG, Bhatt D, Taccioli C, Croce CM (2007) MicroRNA expression patterns to differentiate pancreatic adenocarcinoma from normal pancreas and chronic pancreatitis. JAMA 297(17): 1901-1908.

Bold RJ, Chandra J, McConkey DJ (1999) Gemcitabine-induced programmed cell death (apoptosis) of human pancreatic carcinoma is determined by Bcl-2 content. Ann Surg Oncol 6(3): 279-285.

Bold RJ, Virudachalam S, McConkey DJ (2001) BCL2 expression correlates with metastatic potential in pancreatic cancer cell lines. Cancer 92(5): $1122-1129$.

Borner C, Monney L (1999) Apoptosis without caspases: an inefficient molecular guillotine? Cell Death Differ 6(6): 497-507.

Calin GA, Sevignani C, Dumitru CD, Hyslop T, Noch E, Yendamuri S, Shimizu M, Rattan S, Bullrich F, Negrini M, Croce CM (2004) Human microRNA genes are frequently located at fragile sites and genomic regions involved in cancers. Proc Natl Acad Sci USA 101(9): 2999-3004.

Campbell PM, Lee KM, Ouellette MM, Kim HJ, Groehler AL, Khazak V, Der CJ (2008) Ras-driven transformation of human nestin-positive pancreatic epithelial cells. Methods Enzymol 439: 451-465.

Cande C, Vahsen N, Garrido C, Kroemer G (2004) Apoptosis-inducing factor (AIF): caspase-independent after all. Cell Death Differ 11(6): 591-595.

Deshmukh SK, Srivastava SK, Bhardwaj A, Singh AP, Tyagi N, Marimuthu S, Dyess DL, Zotto VD, Carter JE, Singh S (2015) Resistin and interleukin-6 exhibit racially-disparate expression in breast cancer patients, display molecular association and promote growth and aggressiveness of tumor cells through STAT3 activation. Oncotarget 6(13): 11231-11241.

Elkholi R, Renault TT, Serasinghe MN, Chipuk JE (2014) Putting the pieces together: How is the mitochondrial pathway of apoptosis regulated in cancer and chemotherapy? Cancer Metab 6: 2-16.

Elmore S (2007) Apoptosis: a review of programmed cell death. Toxicol Pathol 35(4): 495-516.

Feldmann G, Maitra A (2008) Molecular genetics of pancreatic ductal adenocarcinomas and recent implications for translational efforts. $J \mathrm{Mol}$ Diagn 10(2): 111-122.

Friedlander MR, Lizano E, Houben AJ, Bezdan D, Banez-Coronel M, Kudla G, Mateu-Huertas E, Kagerbauer B, Gonzalez J, Chen KC, LeProust EM, Marti E, Estivill X (2014) Evidence for the biogenesis of more than 1,000 novel human microRNAs. Genome Biol 15(4): R57-15.

Guo Y, Ziesch A, Hocke S, Kampmann E, Ochs S, De Toni EN, Goke B, Gallmeier E (2015) Overexpression of heat shock protein 27 (HSP27) increases gemcitabine sensitivity in pancreatic cancer cells through S-phase arrest and apoptosis. J Cell Mol Med 19(2): 340-350.

Gupta S, Kass GE, Szegezdi E, Joseph B (2009) The mitochondrial death pathway: a promising therapeutic target in diseases. J Cell Mol Med 13(6): 1004-1033.

Harris MH, Thompson CB (2000) The role of the Bcl-2 family in the regulation of outer mitochondrial membrane permeability. Cell Death Differ 7(12): 1182-1191.

Lee EJ, Gusev Y, Jiang J, Nuovo GJ, Lerner MR, Frankel WL, Morgan DL, Postier RG, Brackett DJ, Schmittgen TD (2007) Expression profiling 
identifies microRNA signature in pancreatic cancer. Int J Cancer 120(5): 1046-1054.

Loukopoulos P, Shibata T, Katoh H, Kokubu A, Sakamoto M, Yamazaki K, Kosuge T, Kanai Y, Hosoda F, Imoto I, Ohki M, Inazawa J, Hirohashi S (2007) Genome-wide array-based comparative genomic hybridization analysis of pancreatic adenocarcinoma: identification of genetic indicators that predict patient outcome. Cancer Sci 98(3): 392-400.

$\mathrm{Lu}$ J, Clark AG (2012) Impact of microRNA regulation on variation in human gene expression. Genome Res 22(7): 1243-1254.

Pogribny IP, Filkowski JN, Tryndyak VP, Golubov A, Shpyleva SI, Kovalchuk O (2010) Alterations of microRNAs and their targets are associated with acquired resistance of MCF-7 breast cancer cells to cisplatin. Int J Cancer 127(8): 1785-1794.

Ryan BM, Robles AI, Harris CC (2010) Genetic variation in microRNA networks: the implications for cancer research. Nat Rev Cancer 10(6): 389-402.

Scherr M, Elder A, Battmer K, Barzan D, Bomken S, Ricke-Hoch M, Schroder A, Venturini L, Blair HJ, Vormoor J, Ottmann O, Ganser A, Pich A, HilfikerKleiner D, Heidenreich O, Eder M (2014) Differential expression of miR$17 \sim 92$ identifies BCL2 as a therapeutic target in BCR-ABL-positive B-lineage acute lymphoblastic leukemia. Leukemia 28(3): 554-565.

Siegel RL, Miller KD, Jemal A (2015) Cancer statistics, 2015. CA Cancer J Clin 65(1): 5-29.

Sperandio S, de Belle I, Bredesen DE (2000) An alternative, nonapoptotic form of programmed cell death. Proc Natl Acad Sci USA 97(26): 14376-14381.

Srivastava SK, Arora S, Singh S, Bhardwaj A, Averett C, Singh AP (2014) MicroRNAs in pancreatic malignancy: progress and promises. Cancer Lett 347(2): $167-174$.

Srivastava SK, Bhardwaj A, Leavesley SJ, Grizzle WE, Singh S, Singh AP (2013) MicroRNAs as potential clinical biomarkers: emerging approaches for their detection. Biotech Histochem 88(7): 373-387.

Srivastava SK, Bhardwaj A, Singh S, Arora S, McClellan S, Grizzle WE, Reed E, Singh AP (2012) Myb overexpression overrides androgen depletioninduced cell cycle arrest and apoptosis in prostate cancer cells, and confers aggressive malignant traits: potential role in castration resistance. Carcinogenesis 33(6): 1149-1157.

Srivastava SK, Bhardwaj A, Singh S, Arora S, Wang B, Grizzle WE, Singh AP (2011) MicroRNA-150 directly targets MUC4 and suppresses growth and malignant behavior of pancreatic cancer cells. Carcinogenesis 32(12): 1832-1839.

Sun CY, Wang BL, Hu CQ, Peng RY, Gao YB, Gu QY, Wang DW (2002) Expression of the bcl-2 gene and its significance in human pancreatic carcinoma. Hepatobiliary Pancreat Dis Int 1(2): 306-308.

Susin SA, Zamzami N, Castedo M, Hirsch T, Marchetti P, Macho A, Daugas E, Geuskens M, Kroemer G (1996) Bcl-2 inhibits the mitochondrial release of an apoptogenic protease. J Exp Med 184(4): 1331-1341.

Susin SA, Zamzami N, Kroemer G (1998) Mitochondria as regulators of apoptosis: doubt no more. Biochim Biophys Acta 1366(1-2): 151-165.

Szafranska AE, Davison TS, John J, Cannon T, Sipos B, Maghnouj A, Labourier E, Hahn SA (2007) MicroRNA expression alterations are linked to tumorigenesis and non-neoplastic processes in pancreatic ductal adenocarcinoma. Oncogene 26(30): 4442-4452.

Tait SW, Green DR (2010) Mitochondria and cell death: outer membrane permeabilization and beyond. Nat Rev Mol Cell Biol 11(9): 621-632.

Tang JT, Wang JL, Du W, Hong J, Zhao SL, Wang YC, Xiong H, Chen HM, Fang JY (2011) MicroRNA 345, a methylation-sensitive microRNA is involved in cell proliferation and invasion in human colorectal cancer. Carcinogenesis 32(8): 1207-1215.

Tyagi N, Bhardwaj A, Singh AP, McClellan S, Carter JE, Singh S (2014) p-21 activated kinase 4 promotes proliferation and survival of pancreatic cancer cells through AKT- and ERK-dependent activation of NF-kappaB pathway. Oncotarget 5(18): 8778-8789.

Yang J, Liu X, Bhalla K, Kim CN, Ibrado AM, Cai J, Peng TI, Jones DP, Wang X (1997) Prevention of apoptosis by Bcl-2: release of cytochrome c from mitochondria blocked. Science 275(5303): 1129-1132.

Zearo S, Kim E, Zhu Y, Zhao JT, Sidhu SB, Robinson BG, Soon PS (2014) MicroRNA-484 is more highly expressed in serum of early breast cancer patients compared to healthy volunteers. BMC Cancer 14: $200-214$.

This work is published under the standard license to publish agreement. After 12 months the work will become freely available and the license terms will switch to a Creative Commons AttributionNonCommercial-Share Alike 4.0 Unported License.

Supplementary Information accompanies this paper on British Journal of Cancer website (http://www.nature.com/bjc) 